\title{
Role of Immunostaining in Detecting Extra-Pattern and Subtle Lymphomatous Infiltration in Bone Marrow Biopsies of NHL Patients
}

\author{
Salwa S. Khodeir, Tahany A. Elkerdany, Abeer Saad Attia, Yasmin N. El Sakhawy, \\ Gehan M. Hamed, Noha Bassiouny Hassan*
}

Department of clinical Pathology, Faculty of Medicine, Ain Shams University Hospital, Cairo, Egypt Email: ^nohabassiouny@med.asu.edu.eg, ^drnohabassiouny@gmail.com, Yasminnabil@hotmail.com, Yasminnabil@med.asu.edu.eg, gehanzeina@yahoo.com

How to cite this paper: Khodeir, S.S., Elkerdany, T.A., Attia, A.S., El Sakhawy, Y.N., Hamed, G.M. and Hassan, N.B. (2018) Role of Immunostaining in Detecting Extra-Pattern and Subtle Lymphomatous Infiltration in Bone Marrow Biopsies of NHL Patients. Open Journal of Blood Diseases, 8, 27-36.

https://doi.org/10.4236/ojbd.2018.82004

Received: May 11, 2018

Accepted: June 23, 2018

Published: June 26, 2018

Copyright $\odot 2018$ by authors and Scientific Research Publishing Inc. This work is licensed under the Creative Commons Attribution International License (CC BY 4.0).

http://creativecommons.org/licenses/by/4.0/

(c) (i) Open Access

\begin{abstract}
Introduction: Immunohistochemistry (IHC) enables the examination of a greater number of trephine biopsy levels and is helpful in determining additional scattered malignant cells. The aim of this study is to detect extra-pattern and subtle lymphomatous infiltration in bone marrow biopsies using CD20 and CD3 immunostaining. Patients and Methods: This study was conducted on 100 newly diagnosed Non Hodgkin Lymphoma (NHL) patients. Their bone marrow trephine biopsies were assessed on routine histology [Hematoxylin and Eosin ( $\mathrm{H} \& \mathrm{E})$ ], and were further subjected to IHC using CD20 and CD3. Results: Pattern of involvement by $\mathrm{H} \& \mathrm{E}$ was highlighted by IHC. It showed additional interstitial pattern in 9 cases, parasinusoidal streaks in one case and highlighted a patchy pattern in another case with interstitial involvement on $\mathrm{H} \& \mathrm{E}$. IHC also detected subtle infiltrations on additional $5.5 \%$ cases compared with histology alone. It helped in differentiating reactive (12 cases) and malignant lymphoid infiltration (33 cases). Conclusion: CD20 and CD3 immunostaining performed routinely on bone marrow trephine biopsies has the ability to reveal extra-pattern of infiltration and improve detection of subtle lymphoid involvement. A combined procedure identifying several distinctive features, in particular histotopography and IHC, provides a promising way of discriminating reactive from neoplastic lymphoid infiltrates in bone marrow trephine biopsies.
\end{abstract}

\section{Keywords}

Immunohistochemistry (IHC), Hematoxylin and Eosin ( $\mathrm{H} \& \mathrm{E})$, Non-Hodgkin Lymphoma (NHL) 


\section{Introduction}

The bone marrow (BM) biopsy is a necessary procedure for patients with lymphomatous neoplasm (NHL) and it's used for staging and following up purposes and hence has an impact on their prognosis and treatment options [1]. It serves occasionally as a primary diagnostic technique in suspicious clinical settings as those with isolated splenomegaly or inaccessible lymphadenopathy [2]. Five major patterns of BM infiltration can be discerned, namely a nodular (intertrabecular or paratrabecular), an interstitial, a diffuse and an intrasinusoidal pattern. However patterns can change during the course of the disease, and mixed patterns occur frequently [3]. Lymphocyte aggregates have been reported to occur in between $1 \%$ and $60 \%$ of normal BM specimens. Therefore, neoplastic lymphomatous infiltrates need to be differentiated from benign (reactive) lymphocytes [4] [5]. Many studies have focused on the use of immunohistochemistry (IHC) on BM trephines in ambiguous cases to differentiate between benign and malignant lymphoid aggregates [6] [7]. IHC enables the examination of a greater number of trephine biopsy levels and is helpful in determining additional scattered and clusters of malignant cells. There are exceptional cases that need IHC to detect subtle lymphomatous cells which might be easily missed if only hematoxylin and eosin ( $\mathrm{H} \& \mathrm{E})$ sections are examined as occult interstitial involvement which usually occur early in the disease course or after treatment as residual cells, intrasinusoidal pattern and accompanying hemophagocytic syndrome [8]. Detection of subtle lymphomatous BM involvement hinges on adequacy of both routine ( $\mathrm{H} \& \mathrm{E})$ histological diagnosis and IHC analysis [3]. This study aimed to reveal extra-pattern and subtle lymphomatous infiltration in bone marrow biopsies using a limited panel of immunostaining (CD20 and CD3) in comparison to $\mathrm{H} \& \mathrm{E}$ sections, and differentiate benign versus malignant lymphoid infiltrates.

\section{Patients and Methods}

The present study was conducted on one hundred newly diagnosed NHL patients (according to their lymph node biopsies) attended hematology department of Ain-Shams University Hospital (ASUH) from June 2015 to August 2016. This study was approved by the ethical and moral committee of Faculty of Medicine Ain-Shams University and a verbal informed consent was taken from all patients participating in the study. They were further classified according to their lymph node biopsy into 94 with B-NHL while 6 patients with T-NHL, their median age was 49 years, 45 of them were males and 55 were females. For staging purposes, bone marrow biopsies were performed with a mean length of $20 \mathrm{~mm}$ and a range of $8-30 \mathrm{~mm}$, in addition to the following.

\subsection{BM Histology}

Routinely, all trephines were fixed in buffered formalin $10 \%$ for 24 hours; decalcification was performed using EDTA for 48 hours. Samples were embedded in 
paraffin, and an attempt was made to stain 5 sections with $\mathrm{H}$ \& E. Sections with at least 5 well preserved marrow spaces were studied for cellularity, normal hematological elements, presence of lymphoid infiltrates if any; the extent and histological pattern were looked for. All specimens were subjected to appropriate IHC for highlighting the pattern and extent of any lymphomatous infiltration, characterizing their nature, differentiating between benign and malignant lymphomatous infiltration, in addition to detecting any subtle infiltrates.

\subsection{IHC}

IHC analysis was performed manually for all cases on formalin fixed, paraffin embedded trephine biopsies. Polymeric HRP (horseradish peroxidase) method was used to immunostain sections using the following monoclonal (mouse ready to use) antibodies: T-cell marker; CD3 (Genemed Biotechnologies, Inc. Code 60-0011-7-USA) and B-cell marker; CD20 (Genemed Biotechnologies; clone L26), in addition to the detection kit (power stain TM 1.0 poly HRP DAB kit for mouse and rabbit). The slides were immersed in heat retrieval solution (Genemed biotechnologies; citrate buffer, $\mathrm{pH} 6$ ) followed by heating in microwave oven adjusted at 800 watt for 10 minutes ( 2 min for 5 cycles). After cooling of the slides at room temperature, peroxidase block $\left(3 \% \mathrm{H}_{2} \mathrm{O}_{2}\right)$ was applied to cover the specimens for 10 - $15 \mathrm{~min}$. Each primary antibody covered the specimens for $60 \mathrm{~min}$ at room temperature, followed by poly -HRP conjugate for $25 \mathrm{~min}$ and lastly DAB solution for $5-10 \mathrm{~min}$ in dark place. Between each step, the slides were immersed and washed 3 times by phosphate buffer saline (PBS) with $\mathrm{pH}$ (7.4 \pm 0.2 ). Counterstaining by Mayer's hematoxylin was used for $40 \mathrm{sec}$ if using stock solution or for 3 - 5 min if using working solution. Dehydration step ended by immersion in xylene solution for 3 min followed by mounting step using distyrene, a plasticizer, and xylene (DPX) after dryness of xylene on slides and then cover slipped. With each run tonsillar tissue was used as positive control, negative control slide was used from the same tissue block, treated as all specimens except for primary antibody which was replaced by PBS. It is particularly important that the negative control slide is subjected to the same retrieval as primary antibody.

A formal system of reporting was developed and was followed for all monoclonals by 2 hematopathologists as presence or absence, localization in its correct site (membranous or nuclear), percentage of positive cells and pattern of infiltration. Regarding CD20 (membranous staining); the percent positivity should be $>10 \%$ of the neoplastic cells [9], and $\geq 50 \%$ of the cells within an aggregate [10]. For CD3 (membranous and cytoplasmic staining); the percent positivity should be $>20 \%$ of the neoplastic cells [11]. CD3 and CD20 are always interpreted together, where the normal ratio of $\mathrm{T}$ to $\mathrm{B}$-cells in $\mathrm{BM}$ being up to 6:1 [12]. Scattered malignant cells may be more easily discernible on IHC analysis. Such cases were classified positive when the numbers of B cells were increased compared with controls [cases with morphologically normal marrow $(n=6)$ ] 
that were used to create a visual impression of normal amounts of background $\mathrm{T}$ (CD3) and B (CD20) cells [13].

\subsection{Statistical Analysis}

In addition to descriptive analysis, data were analyzed using SPSS version 22.0.1 (International Business Machines Corporation, NEW YORK, 2009) statistical package.

\section{Results}

The one hundred NHL patients were further subdivided according to their bone marrow trephine biopsies $(\mathrm{H} \& \mathrm{E})$ results into: Forty nine cases with obvious lymphoid infiltration, which were further classified by IHC as reactive (10 cases) versus malignant (30 cases) lymphoid infiltration, while two cases showed no infiltration on deeper sections taken for IHC. Fifty one cases with no obvious infiltration by H \& E, while by IHC; 44 of them showed concordant no infiltration as $\mathrm{H} \& \mathrm{E}$ while 5 cases with lymphomatous infiltrates by IHC (subtle). Nine cases could not be classified due to detached IHC sections.

\subsection{Agreement between IHC and H \& E Results among All Cases}

IHC results were available in 91 cases. There was an agreement between $\mathrm{H} \& \mathrm{E}$ and IHC $(\mathrm{K}=0.85)$ concerning final interpretation as $40(44 \%)$ out of 91 cases showed lymphoid infiltration by both H \& E and IHC, while 44 (48.3\%) out of 91 cases showed no lymphoid infiltration by both of them. IHC helped in identifying 5 (5.5\%) additional cases with lymphoid infiltration (2 reactive and 3 malignant lymphomatous infiltrates) that were not seen by routine $\mathrm{H} \& \mathrm{E}$ (Figure 1 ), while $2(2.2 \%)$ out of 91 cases showed lymphoid infiltration by $\mathrm{H} \& \mathrm{E}$ that were not seen on IHC sections (Table $1 \&$ Table 2).

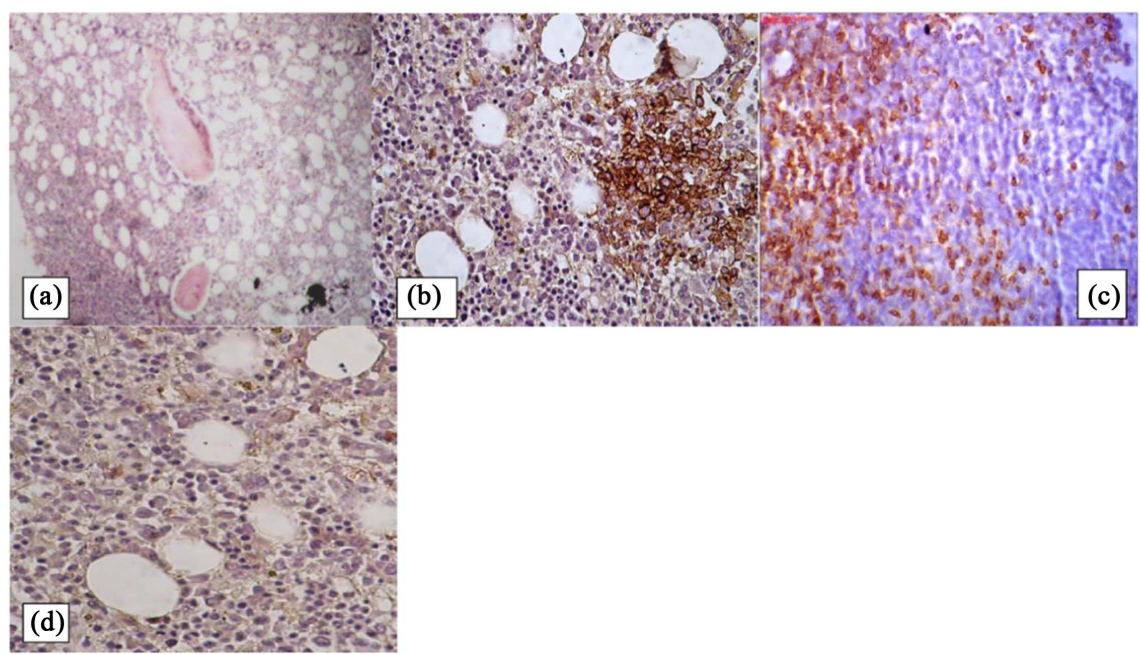

Figure 1. A case of B-NHL showing no obvious lymphoid infiltration in $\mathrm{H} \& \mathrm{E}(\times 10)$. (a) Positive patchy CD20 (×10); (b) Positive interstitial CD20 (×10); (c) Negative CD3 $(\times 10)$; (d) Occult malignant lymphoid infiltration. 
Table 1. Agreement between IHC and H \& E for all cases.

\begin{tabular}{ccccc}
\hline & & \multicolumn{2}{c}{ IHC } & \\
\cline { 2 - 4 } Variable & $\begin{array}{c}\text { Lymphomatous } \\
\text { infiltration }\end{array}$ & $\begin{array}{c}\text { No lymphomatous } \\
\text { infiltration }\end{array}$ & Koreent \\
\hline $\begin{array}{c}\text { Bone marrow } \\
\text { trephine } \\
(\mathrm{H} \& \mathrm{E})\end{array}$ & Not infiltrated & 5 & 44 & $\mathrm{p}$ \\
\hline
\end{tabular}

Table 2. Cases with subtle marrow involvement.

\begin{tabular}{cccccc}
\hline \multirow{2}{*}{ Case No } & H \& E E & \multicolumn{3}{c}{ IHC } & Comment \\
\cline { 3 - 5 } & & CD20 & CD3 & CD20:CD3 & Malignant-infiltration (B-NHL), \\
occult infiltration
\end{tabular}

-: no lymphoid infiltration; +: presence of lymphoid infiltration.

\subsection{Pattern of Infiltration by H \& E versus IHC}

Comparing pattern of lymphoid infiltration between $\mathrm{H} \& \mathrm{E}$ and IHC (Table 3); 10 cases showed diffuse lymphoid infiltration by $\mathrm{H} \& \mathrm{E}$, however by IHC; only $7 / 10$ cases with the same diffuse pattern, $2 / 10$ cases with multiple patchy pattern while the remaining case showed mixed patchy and interstitial pattern. Twenty cases showed patchy infiltration (Figure 2) by routine $\mathrm{H} \& \mathrm{E}$ while by IHC only $10 / 20$ cases with the same pattern of infiltration, where $9 / 20$ cases showed mixed patchy and interstitial infiltration (here IHC by CD20 detected extra-interstitial pattern), and one case showed interstitial pattern. Six cases showed combined patchy and interstitial infiltration by routine $\mathrm{H} \& \mathrm{E}$ which showed same pattern by IHC markers. Four cases showed interstitial infiltration by $\mathrm{H} \& \mathrm{E}, 3 / 4$ cases showed same pattern by IHC (immunostaining by CD20 highlighted para-sinusoidal streaks of lymphomatous infiltration (Figure 3 ) in addition to interstitial pattern in one of those cases), and the remaining one showed patchy infiltration.

\subsection{Significance of Routine Histology (H \& E) and IHC Markers (CD20 \& CD3) in Differentiating Reactive and Malignant Lymphomatous Infiltrates}

There was significant difference regarding pattern of infiltration by $\mathrm{H}$ \& $\mathrm{E}$ 

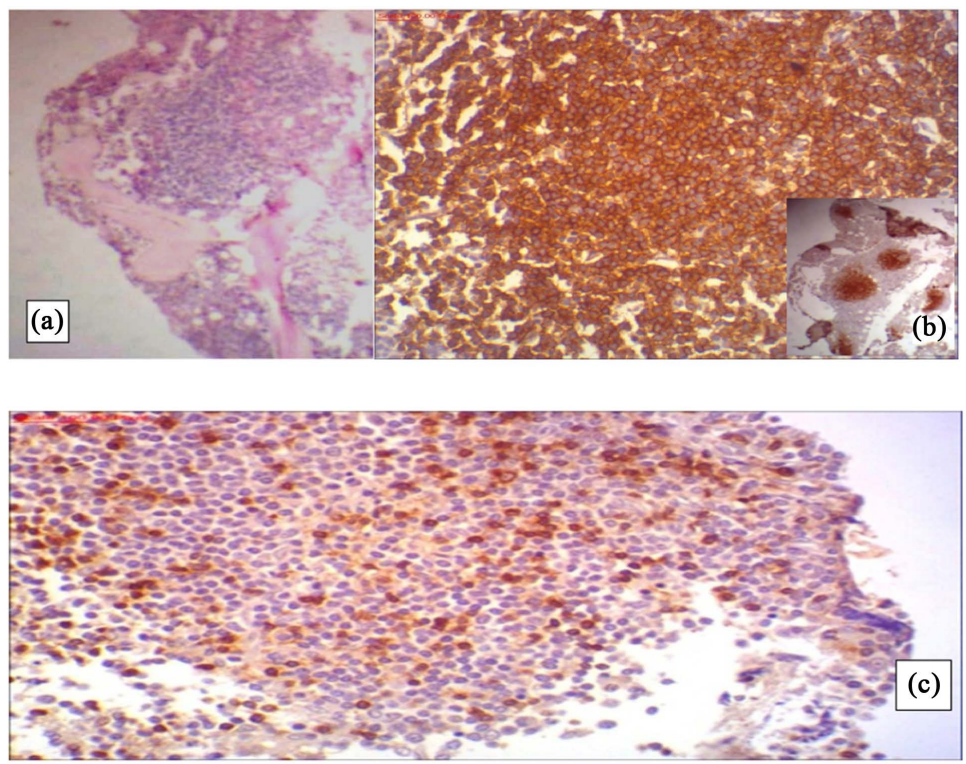

Figure 2. A case of B-NHL showing patchy lymphoid infiltration in $\mathrm{H} \& \mathrm{E}(\times 10)$ : (a) Positive CD20 (×10) (×4); (b) Positive CD3 (×10) (increased CD20: CD3 ratio);

(c) Malignant patchy lymphoid infiltration.

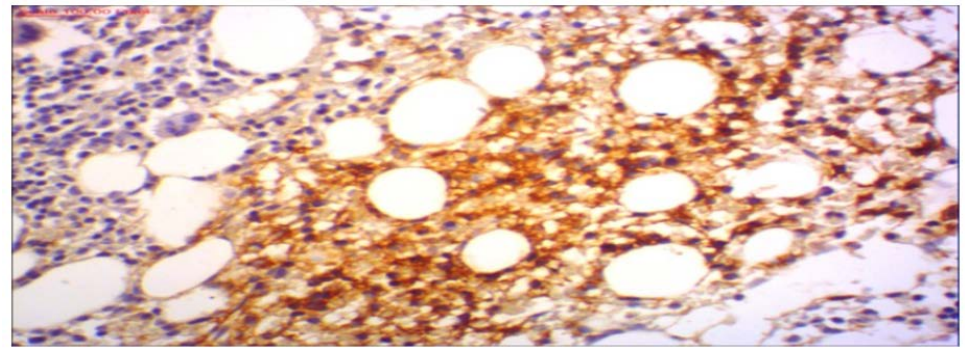

Figure 3. Para-sinusoidal streaks detected by CD20 $(\times 10)$ (extra pattern by IHC).

Table 3. Discrepancy between patterns of infiltration in both H/E versus IHC.

\begin{tabular}{cccccc}
\hline Pattern & Diffuse & Patchy & $\begin{array}{c}\text { Combined patchy } \\
\text { and interstitial }\end{array}$ & Interstitial & No infiltration \\
\hline H \& E & 10 & 20 & 6 & 4 & 49 \\
IHC & 7 & 15 & 16 & 6 & 44 \\
\hline
\end{tabular}

between reactive and malignant lymphomatous infiltrates. Concerning IHC markers used in this study, it was found that CD3 and the ratio between CD20 and CD3 significantly ( $\mathrm{p}=0.001$ ) differentiate between the two types of lymphomatous infiltration together with topographic and morphological appearance.

\section{Discussion}

Small and subtle lymphomatous infiltration of the bone marrow are difficult to diagnose on morphological grounds alone, these infiltrates can be revealed by using IHC and be more extensive than what is apparent on routine $\mathrm{H} \& \mathrm{E}$ stains 
[13]. In our study,CD20 revealed additional interstitial pattern in 9/20 cases which showed patchy pattern of lymphomatous infiltration in $\mathrm{H} \& \mathrm{E}$ sections and also has highlighted a patchy pattern in one case with interstitial infiltration on $\mathrm{H} \& \mathrm{E}$ sections and in another case with interstitial infiltration by $\mathrm{H} \& \mathrm{E}$, immunostaining by CD20 highlighted additionally parasinusoidal streaks of lymphomatous infiltration. This change in pattern of lymphoid infiltration between $\mathrm{H} \& \mathrm{E}$ and IHC was related to the neoplastic cells that are dispersed throughout BM specimen, admixed with haemopoietic cells and may be overlooked by routine $\mathrm{H} \& \mathrm{E}$ and that foci of BM involvement in follicular lymphoma may be subtle, hypocellular or fibrotic, demanding careful scrutiny, prompt sectioning and immunohistochemical procedures [14]. In Thiele et al. [15] study, an ill-defined border of a nodular lymphoid aggregate was recognized with spillage of single lymphocytes between adipocytes or an Indian file like interstitial extension in NHL that can be shown by applying IHC methods. In this study, the use of IHC routinely on all cases detected 5 additional cases (5.5\%) with $\mathrm{BM}$ lymphomatous involvement (3 malignant and 2 reactive infiltrations) that weren't apparent on routine $\mathrm{H} \& \mathrm{E}$ sections. This was lower than the previous study [3] that showed a more modest $11.1 \%$ rate of occult involvement by IHC and this was related to the use of extended panel of immunostaining using two B-cell markers (CD20 \& CD79a), two T-cell marker (CD3 \& CD45RO), and kappa/lambda light chains in addition to examination of additional sections in their immunostaining. In general there is a paucity of data regarding the role of IHC analysis in picking up histological non apparent infiltrate in BM. Fraga et al. [16] reported that in a case series $(n=42)$ of anaplastic large cell lymphoma, routine histology detected marrow involvement in $17 \%$ cases. Routine IHC analysis using CD30 detected occult disease in a further $23 \%$ cases, the inability of routine histology to detect low level disease was related to the scarcity of malignant cells among normal hematopoietic cells and the difficulty in distinguishing them from immature hemopoietic cells

Obvious lymphoid infiltrations (49 cases) by $\mathrm{H} \& \mathrm{E}$ were readily highlighted with the used IHC markers that characterize their nature, histotopography and helped in differentiating them into 12 reactive cases versus 33 cases with malignant lymphoid infiltration. Similarly in a previous study [15], focal lymphoid aggregates in bone marrow trephine biopsies were found in 491 cases where 352 cases were reactive lymphoid nodules and 139 cases of malignant lymphomas based on underlying clinical conditions and marrow features, including in particular histotopography, content of reticulin fibers and IHC using B and T cell markers (CD20, CD45R, CD45RO, CD3). In our study, the ratio between CD20 and CD3 was used to differentiate benign and malignant lymphomatous infiltration, together with their morphological and topographic appearance. Normal ratio (1:1 up to $1: 6)$ was found in $8(100 \%)$ cases, i.e. all of reactive lymphoid cases, decreased ratio $\mathrm{CD} 20<\mathrm{CD} 3(<1: 6)$ was found in only one $(14.3 \%)$ case (T-NHL), increased ratio CD20 > CD3 (>1:6) in 6 (85.7\%) cases (B-NHL). Similarly, chetty et al. [11] and Jalal et al. [12] found that most of reactive lymphoid 
infiltrations are composed mainly of $\mathrm{CD} 3$ positive $\mathrm{T}$ lymphocytes with small numbers of $\mathrm{CD} 20$ positive $\mathrm{B}$ cells as the normal ratio of $\mathrm{T}$ to $\mathrm{B}$ cells in $\mathrm{BM}$ being up to 6:1, these cells are scattered randomly or arranged as small non paratrabecular aggregates. In a study conducted by Lee et al. [17] found that considerable number of B lineage cases showed heterogeneous patterns with evidence of BM infiltration. Thus, heterogeneous immunohistochemical staining patterns (particularly in B lineage lymphomas) may signify malignant lymphoma cell infiltration into the BM.

On the other hand, a previous study [5] considered these cases with combined CD20 \& CD3 must be interpreted cautiously because a wide variety of reaction patterns can occur with both benign lymphoid aggregates and lymphoma. B cell lymphomas may be accompanied by reactive T-lymphocyte. Therefore, a mix of $\mathrm{B}$ and $\mathrm{T}$ cells or a predominant of $\mathrm{T}$ cells doesn't rule out a B cell lymphoma so, the use of a more extended panel of antibodies including anti-Ig $\kappa$ and anti-Ig $\lambda$ will undoubtedly increase the diagnostic potential but still problematical when using fixed and paraffin wax embedded tissue. Monoclonality of these infiltrates was not established in this study unequivocally by seeking light chain restriction. Monoclonality may present a major argument for NHL infiltrates versus benign lymphatic hyperplasia. This finding should be always interpreted in the context of the other features in making the diagnosis as number of infiltrate in section profile, histotopography and reticulin fibers. IHC uniform pattern with predominant of B or T lymphocytes is consistent with malignant lymphoma in contrast with benign lymphoid aggregates that show polyclonal pattern with mixture of B \& T lymphocytes [15].

\section{Conclusion}

In conclusion, our results have demonstrated that using IHC markers (CD20 and CD3), has highlighted extra-pattern and subtle lymphomatous infiltration, in addition to differentiate benign versus malignant infiltrates.

\section{Conflict of Interest}

The authors have no conflict of interest.

\section{Acknowledgements}

Both professor doctor Salwa Saad and Tahany El-Kerdany had supported the work and helped in results interpretation.

\section{References}

[1] Kremer, M., Quintanilla-Martinez, L., Nahrig, J., Von Schilling, C. and Fend, F. (2005) Immunohistochemistry in Bone Marrow Pathology: A Useful Adjunct for Morphologic Diagnosis. Virchows Archiv, 447, 920-937. https://doi.org/10.1007/s00428-005-0070-8

[2] Viswanatha, D. and Foucar, K. (2003) Hodgkin and Non-Hodgkin Lymphoma Involving Bone Marrow. Seminars in Diagnostic Pathology, 20, 196-210. 
https://doi.org/10.1016/S0740-2570(03)00026-1

[3] Riley, R.S., Williams, D., Ross, M. and Zhao, S. (2009) A Bone Marrow Aspirate and Biopsy: A Pathologist's Perspective. Interpretation of the Bone Marrow Aspirate and Biopsies. Journal of Clinical Laboratory Analysis, 23, 259-307. https://doi.org/10.1002/jcla.20305

[4] Dominis, M., Pešut, A., Boroveèki, A., Marušiæ-Vrsaloviæ, M. and Kušec, R. (2005) Bone Marrow Lymphoid Aggregates in Malignant Lymphomas. Croatian Medical Journal, 46, 410-416.

[5] Maes, B., Achten, R., Demunter, A., Peeters, B., Verhoef, G. and De Wolf-Peeters, C. (2000) Evaluation of B Cell Lymphoid Infiltrates in Bone Marrow Biopsies by Morphology, Immunohistochemistry, and Molecular Analysis. Journal of Clinical Pathology, 53, 835-840. https://doi.org/10.1136/jcp.53.11.835

[6] Palacio, C., Acebedo, G., Navarrete, M., Ruiz-Marcellan, C., Sanchez, C., Blanco, A., et al. (2001) Flow Cytometry in the Bone Marrow Evaluation of Follicular and Diffuse Large B-Lymphomas. Haematologica, 86, 934-940.

[7] Mazur, G., Halon, A., Wrobel, T., Jelen, M. and Kuliczkowski, K. (2004) Contribution of Flow Cytometric Immunophenotyping and Bone Marrow Trephine Biopsy in the Detection of Lymphoid Bone Marrow Infiltration in Non-Hodgkin's Lymphomas. Neoplasma, 51, 159-163.

[8] Treetipsatit, J. (2013) Is Immunohistochemistry Mandatory in Staging Bone Marrow Trephine Biopsy of Patients with Diffuse Large B-Cell Lymphoma (DLBCL)? Asian Archives of Pathology, 9, 79-80.

[9] Chu, P.G., Loera, S., Huang, Q. and Weiss, L.M. (2006) Lineage Determination of CD20-B-Cell Neoplasms: An Immunohistochemical Study. American Journal of Clinical Pathology, 126, 534-544. https://doi.org/10.1309/3WG32YRAMQ7RB9D4

[10] West, R.B., Warnke, R.A. and Natkunam, Y. (2002) The Usefulness of Immunohistochemistry in the Diagnosis of Follicular Lymphoma in Bone Marrow Biopsy Specimens. American Journal of Clinical Pathology, 117, 636-643. https://doi.org/10.1309/W3QX-WJ1C-WG2K-225V

[11] Chetty, R., Echezarreta, G., Comley, M. and Gatter, K. (1995) Immunohistochemistry in Apparently Normal Bone Marrow Trephine Specimens from Patients with Nodal Follicular Lymphoma. Journal of Clinical Pathology, 48, 1035-1038. https://doi.org/10.1136/jcp.48.11.1035

[12] Jalal, S.M., Law, M.E., Stamberg, J., Fonseca, R., Seely, J.R., Myers, W.H., et al. (2001) Detection of Diagnostically Critical, Often Hidden, Anomalies in Complex Karyotypes of Haematological Disorders Using Multicolour Fluorescence in Situ Hybridization. British Journal of Haematology, 112, 975-980. https://doi.org/10.1046/j.1365-2141.2001.02630.x

[13] Talaulikar, D., Shadbolt, B., Dahlstrom, J.E. and McDonald, A. (2009) Routine Use of Ancillary Investigations in Staging Diffuse Large B-Cell Lymphoma Improves the International Prognostic Index (IPI). Journal of Hematology \& Oncology, 2, 49-58. https://doi.org/10.1186/1756-8722-2-49

[14] Anagnostou, D. (2005) Pitfalls in the Pattern of Bone Marrow Infiltration in Lymphoproliferative Disorders. Current Diagnostic Pathology, 11, 170-179. https://doi.org/10.1016/j.cdip.2005.01.005

[15] Thiele, J., Zirbes, T.K., Kvasnicka, H.M. and Fischer, R. (1999) Focal Lymphoid Aggregates in Bone Marrow Biopsies: Differentiation between Benign Hyperplasia and Malignant Lymphoma-A Practical Guideline. Journal of Clinical Pathology, 52, 294-300. https://doi.org/10.1136/jcp.52.4.294 
[16] Fraga, M., Brousset, P., Schlaifer, D., Payen, C., Robert, A., Rubie, H., et al. (1995) Bone Marrow Involvement in Anaplastic Large Cell Lymphoma. Immunohistochemical Detection of Minimal Disease and Its Prognostic Significance. American Journal of Clinical Pathology, 103, 82-89. https://doi.org/10.1093/ajcp/103.1.82

[17] Lee, E., Kim, M.Y., Kim, H.S., Kang, H.J., Kim, H.J., Min, S.K., et al. (2017) CD3 and CD20 Immunohistochemical Staining Patterns of Bone Marrow-Infiltrating Malignant Lymphoma Cells. Ann Clin Lab Sci, 47, 136-143. 